BULL. AUSTRAL. MATH. SOC.

VOL. 2 (1970), 138-139.

\title{
Fractional iteration of functions of two variables
}

\section{Phil Diamond}

Let $X \in\left\{R^{2}, C^{2}\right\}$ and

$$
T: x_{1}=\sum a_{k} x^{x^{k} y^{2}}, \quad y_{1}=\sum b_{k} \tau^{x^{k} y^{2}} \quad(k, \tau \geq 0, \quad k+\tau \geq 1)
$$

be an invertible holomorphic function from a neighbourhood of $0 \in X$ to $X$. Denote by $A=\left(\begin{array}{ll}a_{10} & a_{01} \\ b_{10} & b_{01}\end{array}\right)$ the linear part of $T$. In this thesis, the fractional iteration of $T$ is examined when

(1) $X=C^{2}, A=\left(\begin{array}{ll}a & 0 \\ 0 & b\end{array}\right), 0<|b| \leq|a|<1$,

(2) $X=\mathrm{C}^{2}, A=\left(\begin{array}{ll}a & 1 \\ 0 & a\end{array}\right), 0<|a|<1$,

(3) $X=\mathrm{R}^{2}, A=\left(\begin{array}{ll}0 & 1 \\ a & b\end{array}\right), b^{2}<4 a, 0<a<1$,

(4) $X=\mathrm{R}^{2}, \quad A=\left(\begin{array}{ll}1 & 1 \\ 0 & 1\end{array}\right)$.

In the first three cases the fractional iterates are obtained from the algorithm

$$
T(s)=\lim _{n \rightarrow \infty} T^{n} \circ B^{s} \circ T^{n}
$$

where $B$ is the linear or almost linear part of a suitable normal form of $T$.

In case 4, an asymptotic expansion is obtained for the natural iterates $T^{n}$ of higher order near the fixpoint 0 , and this leads to an algorithmic solution of the functional equations

$$
\lambda(T x)=\lambda(x)-1, \mu(T x)=\mu(x), x \in X .
$$

Received 25 June 1969. Thesis submitied to the University of New South Wales, August 1968. Degree approved, February 1969. Supervisor: Professor G. Szekeres. 
The fractional iterates are obtained by solving for $T(s)$ the (invertible) equations

$$
\lambda(T(s) x)=\lambda(x)-s, \mu(T(s) x)=\mu(x), \quad x \in X .
$$

